\title{
Thermodynamic Properties of Liquids, Including Solutions. IX. Thermodynamic Properties of Polymer Solutions*
}

\author{
Maurice L. HugGins \\ Arcadia Institute for Scientific Research, 135 Northridge Lane, \\ Woodside, California 94062, U.S.A.
}

(Received August 9, 1972)

\begin{abstract}
The writer's new theoretical approach to polymer solution thermodynamics is outlined and the results of its application to four types of polymer solutions (rubber-benzene, polyisobutylene-benzene, polyisobutylene-cyclohexane, poly(propylene oxide)- $\mathrm{CCl}_{4}$ ) are presented and briefly discussed. The parameters of the theory are precisely related to the compositions, properties and interactions of the chemical units (segments) in the component molecules. The enthalpy part of the interaction parameter, $\chi_{h}$, depends on an equilibrium between the different types of contact between segment "surfaces." The entropy part of $\chi$ is the sum of a correction for nonrandomness of distribution of the intersegment contacts and a contribution measuring the concentration dependence of the randomness of segment orientations and motions.

KEY WORDS Thermodynamics / Polymer Solutions / Interaction Parameter / Rubber / Polyisobutylene / Poly(propylene oxide) /
\end{abstract}

It is now over 31 years since I first reported ${ }^{9,10}$ on a new theory of the thermodynamic properties of polymer solutions - a theory that has become known by the hyphenated name "FloryHuggins." (Flory ${ }^{11}$ arrived at essentially the same result, publishing his treatment soon after I had reported mine.)

There has been some progress since that time, but I have long considered the theoretical situation very unsatisfactory - both for mixtures of substances of low molecular weight and for high polymer solutions. The Flory-Huggins equation was derived for very dilute solutions. The interaction parameter, $\chi$, in that equation must approach constancy as infinite dilution is approached, but it often varies greatly at higher concentrations, and there has been no good theory to explain and predict these variations. Moreover, there has been no good theory relating the magnitude of $\chi$, even at infinite dilution, to the chemical compositions and properties of the component molecules. Thus it has been impossible to make quantitatively correct predictions about solubilities, solvent

* Presented at the Tokyo Meeting of the Society of Polymer Science, Japan, May 24, 1972. For the previous papers in this series, see ref $1-8$. and plasticizer vapor pressures, equilibria between two liquid phases or between a liquid phase and a gel phase, etc.

So, recently I have been making a new theoretical approach - one that shows promise of eliminating these deficiencies.

It would be especially nice if the parameters in the equations for polymer solutions could be evaluated from measurements on the pure components or on mixtures of simple compounds of low molecular weight. Also, since many polymer and solvent molecules are not chemically uniform but are composed of segments that must affect the thermodynamic properties differently, it would be nice to have parameters characteristic of these component segments and of the interactions between pairs of segments.

I believe that these goals can be achieved. I am trying to build the framework of my new theory in a way that will make it possible.

If the theory is to be satisfactory for polymer solutions it must also be applicable to small molecule solutions. Much of my work to date has been to derive the basic theoretical equations and then to test them on simple, nonpolymeric solutions for which suitable good experimental data are in the literature. The 
equations have also been tested on a few polymer solutions, with the aid of some simplifying assumptions. Some of the results will be presented and briefly discussed after the essentials of the theory have been outlined.

\section{THERMODYNAMIC BACKGROUND}

The properties with which we are concerned are those that determine the distribution of the components between two phases in equilibrium with each other. These properties include vapor pressure, osmotic pressure, solubility, etc. They are all quantitatively related to the Gibbs free energy $(\boldsymbol{G})$ of the solution and its dependence on composition, conveniently expressed as the partial molal free energies of the components $\left(\bar{G}_{1}, \bar{G}_{2}\right)$ or as their activities $\left(a_{1}, a_{2}\right)$ :

$$
\begin{aligned}
& \overline{\boldsymbol{G}}_{1}=\left(\frac{\partial G}{\partial N_{1}}\right)_{N_{2}}=R T \ln a_{1} \\
& \overline{\boldsymbol{G}}_{2}=\left(\frac{\partial G}{\partial N_{2}}\right)_{N_{1}}=R T \ln a_{2}
\end{aligned}
$$

$R$ is the molal gas constant. $T$ is the Kelvin temperature. $N_{1}$ and $N_{2}$ are the numbers of moles of the components. For simplicity, only two-component solutions are dealt with here.

When two pure components are mixed to form a solution, the molal change in Gibbs free energy is related to the changes in enthalpy $(H)$ and entropy $(S)$ by the equation

$$
\Delta G^{\mathrm{M}}=\Delta H^{\mathrm{M}}-T \Delta S^{\mathrm{M}}
$$

From this,

$$
\begin{aligned}
& \bar{G}_{1}=\bar{H}_{1}-T \bar{S}_{1}=R T \ln a_{1} \\
& \bar{G}_{2}=\bar{H}_{2}-T \bar{S}_{2}=R T \ln a_{2}
\end{aligned}
$$

The concepts of enthalpy and entropy are quite different and, in relating the thermodynamic properties to molecular properties, it is convenient to treat enthalpy and entropy changes separately. To understand the dependence of the Gibbs energy of a solution on its composition, one must understand the dependence of the enthalpy and the entropy on the composition.

On mixing liquid or solid components to form a solution, the change in volume is relatively small, hence, at ordinary pressures, the enthalpy change is practically equivalent to the energy change:

$$
\begin{gathered}
\Delta H^{\mathrm{M}}=\Delta E^{\mathrm{M}}+P \Delta V^{\mathrm{M}} \approx \Delta E^{\mathrm{M}} \\
\bar{H}_{1} \approx \bar{E}_{1} \\
\bar{H}_{2} \approx \bar{E}_{2}
\end{gathered}
$$

The energy of a pure liquid or solution is the sum of the vibrational and rotational energy of the molecules, the potential energy of interaction of closest neighbors, and the energy of interaction of pairs of atoms that are not closest neighbors. It is reasonable to assume that, on mixing the solution components, the sum of the kinetic energies and the sum of the interaction energies between nonclosest neighbors change only slightly. To a close approximation, then, these changes can be neglected; we need consider only the changes in the potential energy of the closest neighbor interactions.

The entropy of a pure liquid or solution measures the randomness of location of the molecules (and their parts, if they are not monatomic). This randomness can be divided, conceptually, into two parts: the randomness of distributing the molecules within the total volume and the randomness of the instantaneous locations of the molecules and their parts relative to their average locations. The latter is related to the vibrational and rotational motions and, for nonspherical molecules, to the "freedom" of orientation of the molecules and molecular segments relative to their neighbors.

Mixing component molecules that are of the same size and chemically so similar that the enthalpy (or energy) change is negligible, the change in vibrational, rotational and orientational randomness would also be expected to be negligible. Then, the molal entropy change would be just that associated with the change in randomness of location of the molecular centers when the molecules $\left(x_{1} N_{1}\right.$ molecules of type 1 and $x_{2} N_{2}$ molecules of type 2) are mixed in a perfectly random manner. In 1941, I (and also, independently, Flory) calculated this randomness change and, from it, the associated entropy change statistically, by hypothetically placing the molecules in a perfectly random manner at the points of a lattice.

The result obtained is the following: 


\section{L. HugGins}

$$
\begin{gathered}
\Delta \bar{S}^{\mathrm{M}}=-R\left(x_{1} \ln x_{1}+x_{2} \ln x_{2}\right) \\
\bar{S}_{1}=-R \ln x_{1} \\
\bar{S}_{2}=-R \ln x_{2}
\end{gathered}
$$

Here and elsewhere, $x_{1}$ and $x_{2}$ designate mole fractions. Substituting into eq 4 and 5 , one finds that, if the heat of mixing $\left(\Delta \bar{H}^{\mathrm{M}}\right)$ is zero,

$$
\begin{aligned}
& a_{1}=x_{1} \\
& a_{2}=x_{2}
\end{aligned}
$$

One thus obtains a theoretical derivation of the originally empirical Raoult's law.

The same procedure was used by me (and by Flory) to calculate the entropy of randomness of location of the molecules and molecular segments for a solution of a chain polymer in a small molecule liquid. $^{9-12}$ Assuming perfect randomness of placement of the molecules and segments, we obtained for very dilute solutions:

$$
\begin{aligned}
& \Delta \bar{S}^{\mathrm{M}}=-R\left(x_{1} \ln \phi_{1}+x_{2} \ln \phi_{2}\right) \\
& \bar{S}_{1}=-R\left[\ln \phi_{1}+\left(1-\frac{\bar{V}_{1}}{\bar{V}_{2}}\right) \phi_{2}\right] \\
& \bar{S}_{2}=-R\left[\ln \phi_{2}+\left(1-\frac{\bar{V}_{2}}{\bar{V}_{1}}\right) \phi_{1}\right]
\end{aligned}
$$

$\phi_{1}$ and $\phi_{2}$ are volume fractions. $\bar{V}_{1}$ and $\bar{V}_{2}$ are partial molal volumes. Their ratio is not appreciably different from the ratio of the molal volumes of the pure liquids.

These equations have been shown to account well for the very large departures from Raoult's law behavior that have been observed for dilute solutions of high polymers in low-molecularweight solvents. They have also supplied a theoretical basis for the determination of polymer molecular weights from appropriate extrapolations of osmotic pressure, light scattering and other data to infinite dilution.

I obtained eq 14-16 later without using the hypothetical lattice. ${ }^{13-16}$ Moreover, as I have recently shown, ${ }^{4}$ practically the same result is obtained for spherical molecules over the whole composition range. One can thus reasonably assume that substitution of eq 14-16 for the Raoult's law eq 9-11 adequately takes care of the effect of relative molecular sizes on the entropy of placement of the molecules in the solution volume, provided the placement is perfectly random. Correction should be made for departures from perfect randomness and allowance should be made for entropy of mixing contributions related to changes (with concentration) of the vibrational, rotational, and orientational entropy.

If the heat of mixing is not negligible, Raoult's law does not hold, even for molecules of the same size. See eq $3-5,12$, and 13. Hildebrand ${ }^{17}$ and others, however, have found it useful to make the approximation that the entropy eq 9-11, on which Raoult's law is based, do hold for many solutions, (which they call "regular solutions"). They calculate the heat of mixing with the aid of some further approximations (e.g., that the cohesive energy density of a pure liquid equals the ratio of its energy of vaporization to its molal volume, and that the interaction energy between two unlike molecules is the geometric mean of the interaction energies between pairs of like molecules). They thus obtain equations for the Gibbs energy of the solution, from which one can compute approximate relative solubilities. For molecules differing greatly in size they have, since the early 1940's, replaced the Raoult's law entropy eq 9-11 by the Flory-Huggins equations.

In my 1941 derivation of the equation for the entropy of solutions of chain molecules, I showed that a term of the form $\chi_{s} \phi_{2}{ }^{2}$ should be added to the expression in the brackets in eq 15:

$$
\bar{S}_{1}=-R\left[\ln \phi_{1}+\left(1-\frac{\bar{V}_{1}}{\bar{V}_{2}}\right) \phi_{2}+\chi_{s}{\phi_{2}}^{2}\right]
$$

$\chi_{s}$ must approach constancy as infinite dilution is approached, but it may vary at higher concentrations.

The Scatchard-Hildebrand treatment of heats of mixing, and other related treatments, require that

$$
\bar{H}_{1}=R T \chi_{h} \phi_{2}{ }^{2}
$$

with the parameter $\chi_{h}$ approaching constancy as infinite dilution is approached, but in general varying at higher concentrations.

From eq 4,17 , and 18 ,

$$
\bar{G}_{1}=R T \ln a_{1}=R T\left[\ln \phi_{1}+\left(1-\frac{\bar{V}_{1}}{\bar{V}_{2}}\right) \phi_{2}+\chi \phi_{2}{ }^{2}\right]
$$


Thermodynamic Properties of Liquids. IX.

with

$$
\chi=\chi_{h}+\chi_{s}
$$

At various times ${ }^{14-16,18}$ I have attempted to relate $\chi$ and its concentration dependence to molecular structures and intermolecular properties, but the relations deduced, until recently, have been fairly complicated and have involved parameters that are not easy to evaluate experimentally.

\section{NEW THEORY}

Most of the ideas underlying my new treatment $I$ have used previously in theorizing about polymer solutions. Most of them have also been used by others, though not in quite the same way. Any attempt to give credit to all whose ideas are incorporated in this new development would necessarily be very lengthy. No attempt will be made here. References to some of the related earlier work by others are included in ref 6 and 7.

I deal first ${ }^{1}$ with the molal enthalpy of mixing, $\Delta \bar{H}^{\mathrm{M}}$, equal to the excess enthalpy, $\bar{H}^{\mathrm{E}}$, and practically equal to the excess energy, $\bar{E}^{\mathrm{E}}$.

I assume that a liquid behaves thermodynamically as if its molecules were each composed of one or more chemically uniform segments, these segments having "surfaces" in mutual contact. For each type of segment $(\alpha, \beta, \ldots)$ the average contacting surface area per mol of segments $\left(\sigma_{\alpha}{ }^{0}, \sigma_{\beta}{ }^{0}, \ldots\right)$ is assumed to be constant at a given temperature. The energy $(\varepsilon)$ per unit area of contact, for each contact type $(\alpha \alpha, \beta \beta$, $\alpha \beta, \ldots)$ is assumed to be constant at a given temperature. The relative total contact areas for the different types are assumed to be governed by equilibrium constants. This minimizes the Gibbs free energy. If the system is ditonic (having just two types of segment) there are three types of contact and

$$
K=\frac{\sigma_{\alpha \beta}^{2}}{4 \sigma_{\alpha \alpha} \sigma_{\beta \beta}}
$$

One might equivalently define $\sigma_{\alpha}{ }^{0}$ and $\sigma_{\beta}{ }^{0}$ as the average numbers of contacts per mol of segment for each of the $\alpha$ and $\beta$ types. $\sigma_{\alpha \alpha}$, $\sigma_{\beta \beta}$, and $\rho_{\alpha \beta}$ would then be average energies per contact.
From these assumptions one can readily deduce equations for $\bar{H}^{\mathrm{E}}$. For the simple case of a ditonic mixture of two monotonic singlesegment molecules,

$$
\begin{aligned}
\bar{H}^{\mathrm{E}}= & \frac{-\left(x_{1} \sigma_{\alpha}{ }^{0}+x_{2} \sigma_{\beta}{ }^{0}\right) \Delta \varepsilon}{K^{\prime}} \\
& \times\left\{1-\left[1+\frac{K^{\prime} x_{1} \sigma_{\alpha}{ }^{0} x_{2} \sigma_{\beta}{ }^{0}}{\left(x_{1} \sigma_{\alpha}{ }^{0}+x_{2} \sigma_{\beta}{ }^{0}\right)^{2}}\right]^{1 / 2}\right\}
\end{aligned}
$$

or

$$
\begin{aligned}
\bar{H}^{\mathrm{E}}= & \frac{-2 \varepsilon_{\Delta}}{K^{\prime}}\left[1+x_{2}\left(r_{\sigma}-1\right)\right] \\
& \times\left\{1-\left[1+\frac{K^{\prime} r_{\sigma}\left(x_{2}-x_{2}{ }^{2}\right)}{\left[1+x_{2}{ }^{2}\left(r_{\sigma}-1\right)\right]^{2}}\right]^{1 / 2}\right\}
\end{aligned}
$$

with

$$
\begin{gathered}
\varepsilon_{\Delta}=\frac{\sigma_{\alpha}{ }^{0} \Delta \varepsilon}{2}=\frac{\sigma_{\alpha}{ }^{0}}{2}\left(2 \varepsilon_{\alpha \beta}-\varepsilon_{\alpha \alpha}-\varepsilon_{\beta \beta}\right) \\
K^{\prime}=4\left(\frac{1}{K}-1\right)
\end{gathered}
$$

and

$$
r_{\sigma}=\sigma_{\beta}{ }^{0} / \sigma_{\alpha}{ }^{0}
$$

For perfectly random mixing of the contacts, $K=1, K^{\prime}=0$, and

$$
\bar{H}^{\mathrm{E}}=\varepsilon_{\Delta}\left(\frac{r_{\sigma} x_{1} x_{2}}{x_{1}+r_{\sigma} x_{2}}\right)=\varepsilon_{\Delta}\left[\frac{r_{\sigma}\left(x_{2}-x_{2}{ }^{2}\right)}{1+x_{2}\left(r_{\sigma}-1\right)}\right]
$$

From eq 23 it is seen that three parameters are required to give the dependence of $\bar{H}^{\mathrm{E}}$ on concentration. One $\left(\varepsilon_{\Delta}\right)$ is a scaling parameter, the second ( $K$ or $K^{\prime}$ ) determines the "fatness" of the curve, and the third $\left(r_{o}\right)$ determines its dissymmetry (skewness). See Figure 1. The last mentioned parameter is not always arbitrary; it must be consistent with other similar parameters involving the same components. Also, all three parameters are characteristic of all ditonic systems containing these same two segment types, although some adjustment must sometimes be made because of steric differences (e.g., those affecting accessibility of the segment surfaces).

Testing these equations on many simple mixtures for which good experimental $\bar{H}^{\mathrm{E}}$ data are available, it has been found that nearly always the data agree with eq 23 within the probable error of the experiments. I shall cite only one example. Figure 2 shows points calculated from 


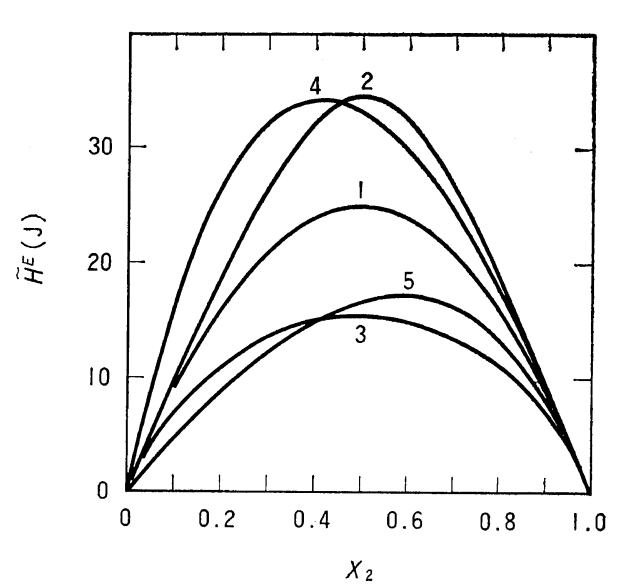

Figure 1. Theoretical dependence of the molal excess enthalpy on mol fraction for mixtures of two substances, each composed of single segment molecules. The same curves apply to any mixture of two monotonic substances, if $H^{\mathrm{E}}$ is for a mol of segments and $x_{2}$ is replaced by $x_{\beta}$, the segment fraction. (Reprinted from ref 6.) For all curves: $\varepsilon_{\Delta}=100 \mathrm{~J}$; curve $1, r_{\sigma}=1, K=1$; curve $2, r_{\sigma}=1, K=5$; curve $3, r_{\sigma}=1, K=0.2$; curve $4, r_{\sigma}=2, K=1$; curve $5, r_{\sigma}=0.5, K=1$.

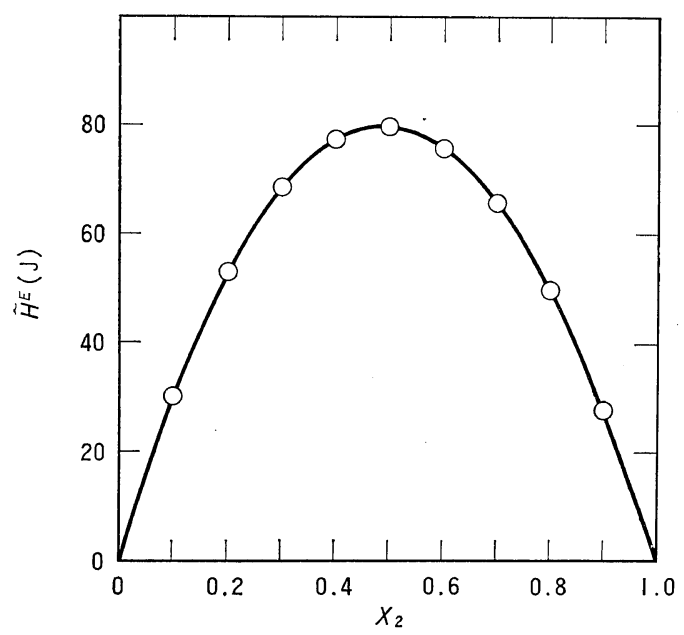

Figure 2. Enthalpy of mixing for $\mathrm{CCl}_{4}-c-\mathrm{C}_{5} \mathrm{H}_{10}$ at $25^{\circ} \mathrm{C}$. Circles are for experimental data by Ewing and Marsh. ${ }^{19}$ The curve is theoretical, for $\varepsilon_{\Delta}=305.075 \mathrm{~J} \mathrm{~mol}^{-1} ; \quad r_{\sigma}=1.12073, \quad K=0.956$. (Reprinted from ref 6. )

Ewing-Marsh's equation (which represents their data to within $0.1 \mathrm{~J} \mathrm{~mol}^{-1}$ ) for carbon tetrachloride-cyclopentane. The agreement between these points and the theoretical curve is within $0.1 \mathrm{~J} \mathrm{~mol} \mathrm{mov}^{-1}$ over the whole composition range.

Theoretical equations, corresponding to eq 23 , for more complex systems are readily deduced. Only slightly more complicated than eq 23 is that for a polymer solution in which each polymer mer is sufficiently chemically homogeneous to be treated as a single segment. For such a ditonic solution, the parameter $\chi_{h}$ is given by the equation

$$
\begin{aligned}
\chi_{h} & =\frac{1}{R T{\phi_{2}}^{2}}\left(\frac{\partial \bar{H}^{\mathrm{E}}}{\partial N_{1}}\right)_{N_{2}} \\
& =\frac{\varepsilon_{\Delta} z_{\beta}}{R T{\phi_{2}}^{2}}\left(\frac{1-z_{\alpha} g_{K}}{1+z_{\alpha} z_{\beta} K^{\prime} g_{K} / 2}\right)
\end{aligned}
$$

where

$$
\begin{gathered}
z_{\beta}=1-z_{\alpha}=\frac{\left(\frac{r_{\sigma}}{r_{v}}\right) \phi_{2}}{1+\left(\frac{r_{\sigma}}{r_{v}}-1\right) \phi_{2}} \\
r_{v}=V_{2} / V_{1}
\end{gathered}
$$

and

$$
g_{K}=\frac{-2}{z_{\alpha} z_{\beta} K^{\prime}}\left[1-\left(1+K^{\prime} z_{\alpha} z_{\beta}\right)\right]
$$

The excess entropy of mixing is defined as the entropy change, per mol, on mixing, minus what it would be if Raoult's law were obeyed. The part of this excess entropy which is due to the difference in the sizes of the molecules, assuming random mixing, can be taken ${ }^{4}$ as the difference between eq 14 and 9:

$$
\bar{S}_{\mathrm{rm}}^{\mathrm{E}}=-R\left[x_{1} \ln \left(\frac{\phi_{1}}{x_{1}}\right)+x_{2} \ln \left(\frac{\phi_{2}}{x_{2}}\right)\right]
$$

If the equilibrium constant $K$ is not unity, the mixing is not perfectly random. The probability that a segment of one type contacts another segment of the same type is not equal to the probability that it contacts a segment of the other type, even when the contacting areas of the two types are equal. I have deduced ${ }^{4}$ an equation for the difference between the "combinatorial entropy" (the entropy of placement of the molecules in the total volume) and what it would be if there were random mixing of the contacts. This combinatorial entropy correction $\left(\bar{S}_{\mathrm{cc}}^{\mathrm{E}}\right)$ is given by the equation 
Thermodynamic Properties of Liquids. IX.

$$
\begin{aligned}
\bar{S}_{\mathrm{cc}}^{\mathrm{E}}= & \frac{-\boldsymbol{R} \sigma_{\alpha}{ }^{*}}{4}\left\{x _ { 1 } \left[\left(1-z_{\beta} g_{K}\right) \ln \left(1-z_{\beta} g_{K}\right)\right.\right. \\
& \left.-z_{\alpha} \ln z_{\alpha}-z_{\beta} \ln \left(z_{\alpha} z_{\beta}\right)\right] \\
& +x_{2} r_{\alpha}\left[\left(1-z_{\alpha} g_{K}\right) \ln \left(1-z_{\alpha} g_{K}\right)\right. \\
& \left.\left.-z_{\beta} \ln z_{\beta}+z_{\alpha} g_{K} \ln \left(z_{\alpha} z_{\beta} g_{K}{ }^{2}\right)\right]\right\}
\end{aligned}
$$

The corresponding contribution to $\chi_{s}$, for a monotonic polymer in a monotonic singlesegment solvent, is

$$
\begin{aligned}
\chi_{s, \mathrm{cc}}= & \frac{-\sigma_{\alpha}{ }^{*}}{4{\phi_{2}}^{2}}\left[\ln \left(\frac{1-z_{\beta} g_{K}}{z_{\alpha}}\right)\right. \\
& +\left(\frac{z_{\beta}-2 z_{\beta}{ }^{2}}{1+K^{\prime} z_{\alpha} z_{\beta} g_{K} / 2}-z_{\alpha} z_{\beta} g_{K}\right) \\
& \left.\times \ln \left(1+\frac{1-g_{K}}{z_{\alpha} z_{\beta} g_{K}{ }^{2}}\right)\right]
\end{aligned}
$$

These combinatorial entropy correction equations contain two parameters, $K$ and $r_{\sigma}$, that can be evaluated from the shapes of the excess enthalpy curves, plus another parameter, $\sigma_{\alpha}{ }^{*}$. This is the number of units of surface area, per mol of $\alpha$ segments, that contact other surface area units independently, in a hypothetical model solution for which the combinatorial entropy is the same as in the actual solution. Alternatively, $\sigma_{\alpha}{ }^{*}$ can be considered to be the number of independent contacts per mol of $\alpha$ segments, in the equivalent hypothetical model solution.

To obtain the vibrational, rotational and orientational randomness contribution ${ }^{4}$ to the entropy of a ditonic system, I make the simple assumption that, for each type of segment, the randomness is a rectilinear function of the fraction of the contacting surface that makes contact with a surface of the other kind. This leads to the equations

$$
\bar{S}_{\mathrm{or}}^{\mathrm{E}}=R x_{2} \ln \left(1+k_{s}{ }^{\prime} z_{\alpha} g_{K}\right)
$$

and

$$
\chi_{s, \mathrm{or}}=\frac{-k_{s}{ }^{\prime} z_{\beta}}{r_{o} \phi_{2}{ }^{2}\left(1+k_{s}{ }^{\prime} z_{\alpha} g_{K}\right)}\left[\frac{z_{\beta}-z_{\alpha}}{\left(1+K^{\prime} z_{\alpha} z_{\beta}\right)^{1 / 2}}+z_{\alpha} g_{K}\right]
$$

These equations, like those for the correction to the combinatorial entropy, involve the ratio $r_{\sigma}$ and the equilibrium constant $K$, which can be determined from the concentration dependence of the excess enthalpy. They also contain a new constant, $k_{s}{ }^{\prime}$, measuring the average change in randomness of a segment when the concentration changes from practically infinite dilution to the pure liquid component, averaged over the two segment types.

Each of the two new types of entropy contribution thus involves one new constant. I do not now see any way of evaluating these, other than from the entropy data for the system of interest or for another system having the same types of segments. These constants have definite theoretical meanings. It is hoped that, after they have been determined for a number of systems, it will become possible to estimate their values for new systems. The constants may also be useful in dealing theoretically with other properties, such as viscosity and longrange elasticity.

\section{APPLICATIONS TO POLYMER SOLUTIONS}

Entropies of mixing are not measured directly, but are computed from Gibbs energies and excess enthalpies, using eq 13. Although I have applied this theory to some mixtures of lowmolecular-weight compounds for which the

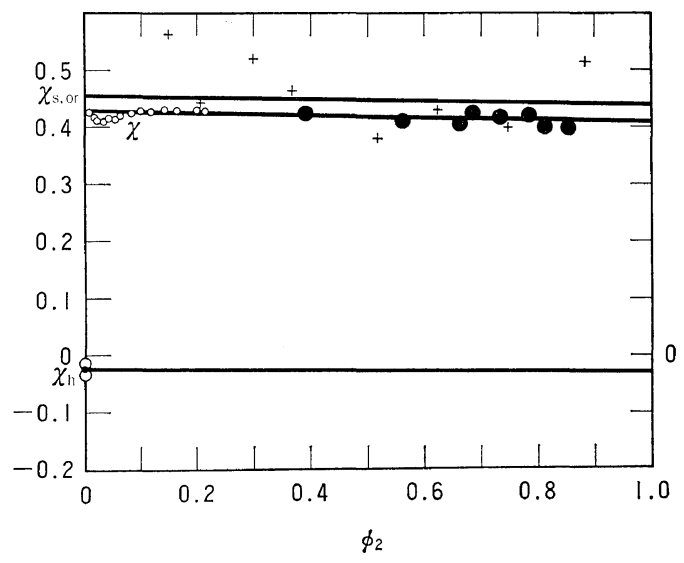

Figure 3. The interaction parameter $(\chi)$ and its components for polyisobutylene-cyclohexane solutions. The curves are theoretical, for $\varepsilon_{\Delta}=-18$ cal $\mathrm{mol}^{-1}, r_{\sigma}=0.51, K=1$, and $k_{s}{ }^{\prime}=-0.22$. Data points for $\chi_{h}$ : $\bigcirc$, Delmas, Patterson, and Somcynsky; ${ }^{20} \bigcirc$, Watters, Daoust, and Rinfret. ${ }^{21}$ Data points for $\chi$ : $O$, Flory and Daoust; ${ }^{22} \bigcirc$, Eichinger and Flory; ${ }^{23}+$, Bawn and Patel. ${ }^{24}$ (Reprinted from ref 7.) 


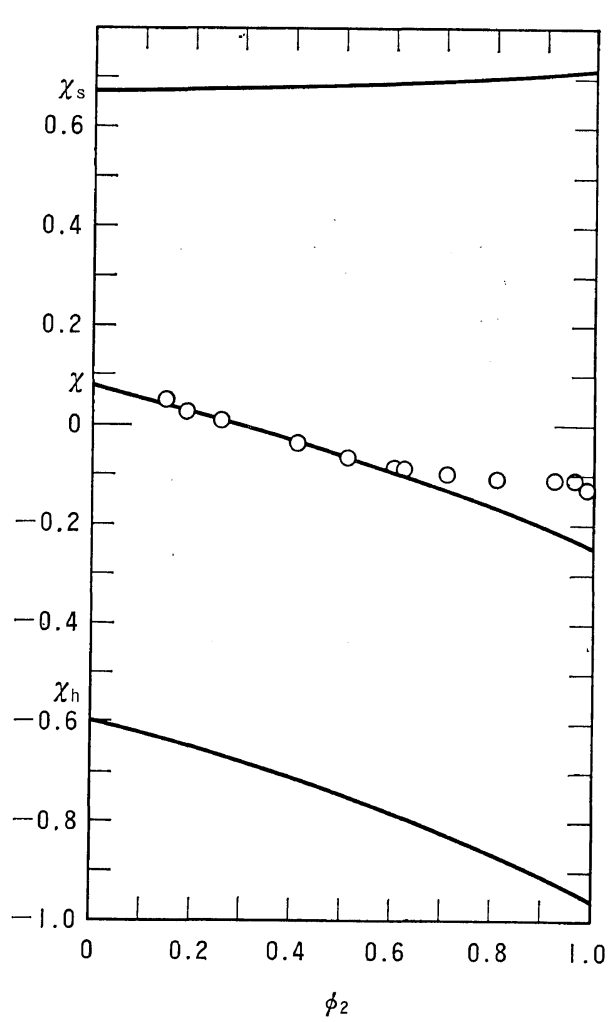

Figure 4. The interaction parameter and its components ( $\chi_{h}$ and $\chi_{s}=\chi_{s}$,or $)$ for solutions of poly(propylene oxide) in $\mathrm{CCl}_{4}$. The curves are theoretical, for $\varepsilon_{\Delta}=-500 \mathrm{cal} \mathrm{mol}^{-1}$ and $k_{s}{ }^{\prime}=-0.36$, $r_{\sigma}=0.54$, and $K=1$. Data by Kershaw and Malcolm. ${ }^{25}$ (Reprinted from ref 5.)

excess entropy is not negligible, the results are not yet ready to be reported. I have, however, made and reported on several studies of polymer solutions, treated as ditonic. In each case the excess entropy, which is the sum of $\bar{S}_{\mathrm{rm}}^{\mathrm{E}}, \bar{S}_{\mathrm{cc}}^{\mathrm{E}}$ and $\bar{S}_{\mathrm{or}}^{\mathrm{E}}$, is far from negligible. Subtraction of $\bar{S}_{\mathrm{rm}}^{\mathrm{E}}$, which is calculable from the molal volumes, leaves $\bar{S}_{\mathrm{cc}}^{\mathrm{E}}+\bar{S}_{\mathrm{or}}^{\mathrm{E}}$. In the polyisobutylene-cyclohexane ${ }^{7}$ and poly(propylene oxide) $-\mathrm{CCl}_{4}$ systems $^{5}$ the enthalpy data show that $K$ must be close to unity, hence $\bar{S}_{\mathrm{cc}}^{\mathrm{E}}$ and $\chi_{s, \mathrm{cc}}$ must be negligible. This leaves only $\bar{S}_{\mathrm{or}}^{\mathrm{E}}$ or $\chi_{s, \mathrm{or}}$, with one parameter, to be determined from the data for the total $\chi$. If that parameter is chosen to give agreement for one concentration, the theory (with the $r_{\sigma}$ and $\varepsilon_{\Delta}$ parameters from the enthalpy data) gives the theoretical curve for the whole concentration range. Figures 3 and 4 show the degree of agreement between the $\chi$ curves obtained in this way and the best experimental data. They also show the contributions of the components of $\chi$. Considering the approximations of the theory and imperfections in the experimental data, I consider the results to be very encouraging.

The enthalpy data for the rubber-benzene $e^{2,3}$ and polyisobutylene-benzene ${ }^{7}$ systems show that $K$ is, in both cases, appreciably different from one. $\chi_{s, \mathrm{cc}}$ is therefore not negligible and two parameters (in addition to those obtained from the enthalpy curves) must be evaluated. This can be done by requiring agreement with the total $\chi$ data at two points. Figures 5 and 6 show the agreement obtained in this way with the experimental $\chi$ values. As for the

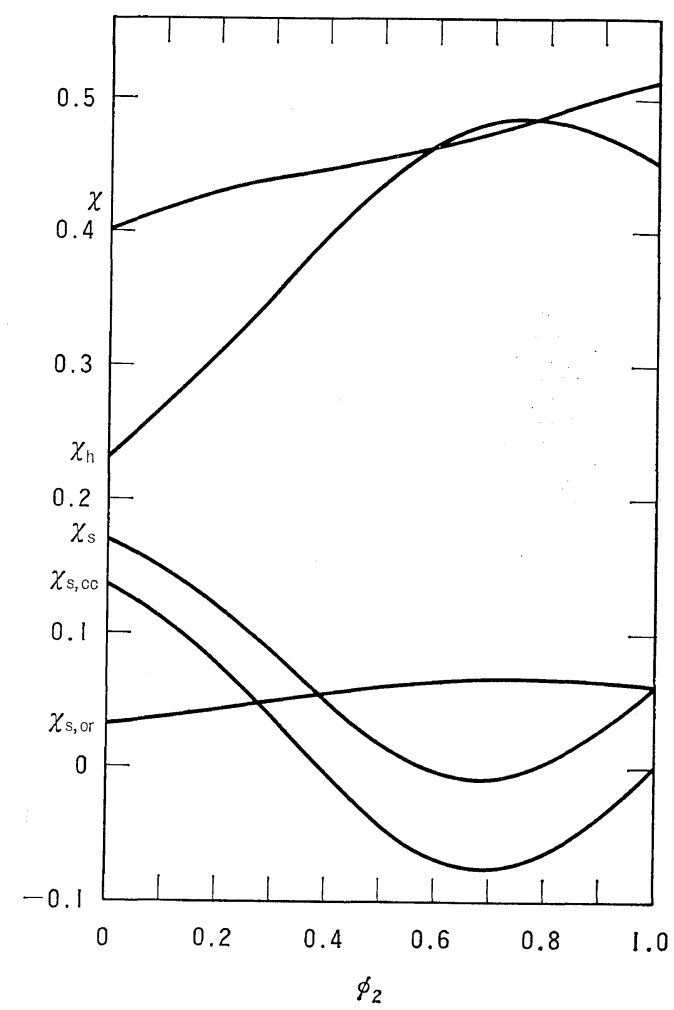

Figure 5. The interaction parameter and its components for rubber-benzene solutions. The curves are theoretical, for $\varepsilon_{\Delta}=268 \mathrm{cal} \mathrm{mol}^{-1}, \quad r_{\sigma}=0.74$, $K=1.54, k_{s}{ }^{\prime}=0.32$, and $\sigma_{\alpha}{ }^{*}=5$. Based on experimental data from Gee and Treloar, ${ }^{26}$ Gee and Orr, ${ }^{27}$ and Eichinger and Flory. ${ }^{28}$ (Reprinted from ref 2.) 


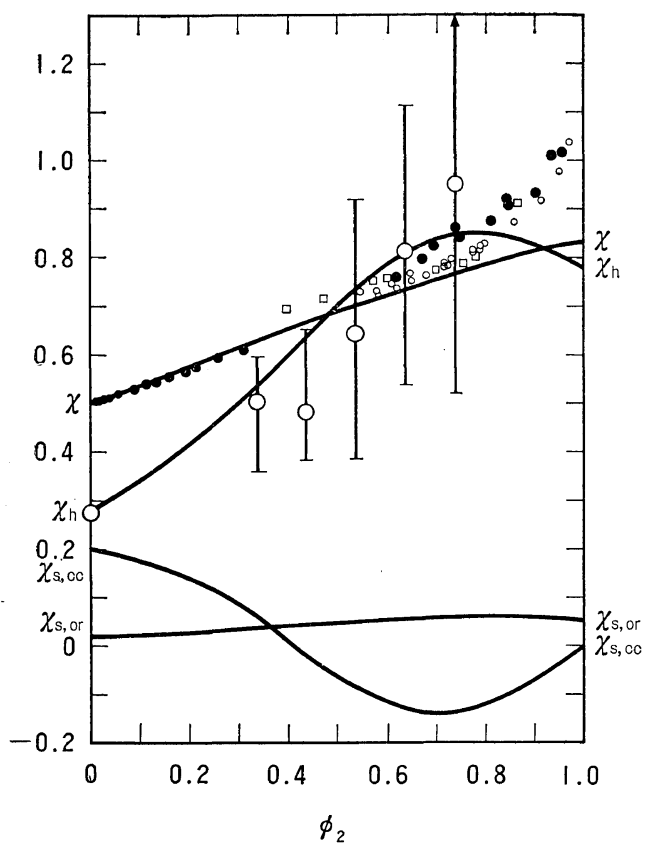

Figure 6. The interaction parameter and its components for polyisobutylene-benzene solutions. The curves are theoretical, for $\varepsilon_{4}=463 \mathrm{cal} \mathrm{mol}^{-1}$, $r_{\sigma}=0.56, K=1.88, k_{s}{ }^{\prime}=-0.03$, and $\sigma_{\alpha}{ }^{*}=9$. Data points for $\chi_{h}$ : $\bigcirc$, Krigbaum and Flory;29 Eichinger and Flory. ${ }^{30}$ Data points for $\chi$ : $\bullet$, Flory and Daoust; ${ }^{22}$, Eichinger and Flory; ${ }^{30}$ O, Jessup; ${ }^{31} \square$, Bawn and Patel. ${ }^{24}$ (Reprinted from ref 7.)

other systems, the curves for the components of $\chi$ are also shown. Note the importance of the entropy components relative to that of the enthalpy component. Also note the very different concentration dependence for the different components. These results show, I believe, that no theory that neglects these entropy factors can successfully reproduce the concentration dependence of the Gibbs energy of polymer solutions; hence, no such theory can hope to be very successful in predicting solubilities and other properties dependent on the Gibbs energies.

\section{ENERGY-VOLUME-ENTROPY RELATIONS}

Unlike other theoretical treatments of solutions now in vague, this one does not assume the same relations between energy, volume and entropy for solutions as for the pure liquids. These properties must depend, not only on the changes in intermolecular (or intersegment) distances, but also (and more importantly) on the changes in types and numbers (or areas) of contacts between neighbors. The dependence of energy on intersegment distances and coordination numbers is of course important in determining the magnitudes of some of the parameters entering into this theory and their temperature dependence. I hope to have a theoretical treatment of this subject ready for publication before long.

\section{SUMMARY}

I have summarized the thermodynamics pertinent to a theory of the thermodynamic properties of solutions. I have outlined the theory as it was until recently and have described my new approach, emphasizing the concepts underlying the equations used. I have given examples of comparisons between theoretical curves and experimental data, showing very encouraging agreement. Obviously, much more remains to be done, but I am hopeful that good agreement will continue to be obtained. I believe that this theory will prove to be useful.

\section{REFFRENCES}

1. M. L. Huggins, J. Phys. Chem., 74, 371 (1970).

2. M. L. Huggins, Polymer, 12, 389 (1971).

3. M. L. Huggins, J. Polym. Sci., Part C, 33, 55 (1971).

4. M. L. Huggins, J. Phys. Chem., 75, 1255 (1971).

5. M. L. Huggins, Macromolecules 4, 274 (1971).

6. M. L. Huggins, J. Paint Technol., 44, 55 (1972).

7. M. L. Huggins, Pure Appl. Chem., 31, 245 (1972).

8. M. L. Huggins, J. Polym. Sci., Part C, in press.

9. M. L. Huggins, J. Chem. Phys., 9, 440 (1941).

10. M. L. Huggins, J. Phys. Chem., 46, 151 (1942).

11. P. J. Flory, J. Chem. Phys., 9, 660 (1941); 10, 51 (1942).

12. M. L. Huggins, Ann. N.Y. Acad. Sci., 43, 1 (1942).

13. M. L. Huggins, J. Phys. Coll. Chem., 52, 248 (1948).

14. M. L. Huggins, J. Polym. Sci., 16, 209 (1955).

15. M. L. Huggins, "Physical Chemistry of High 


\section{L. HugGins}

Polymers," John Wiley \& Sons, Inc., New York, N.Y., 1958.

16. M. L. Huggins, J. Amer. Chem. Soc., 86, 3535 (1964).

17. J. H. Hildebrand and R. L. Scott, "Regular Solutions," Prentice-Hall, Englewood Cliffs, N.J., 1962.

18. M. L. Huggins, Ann. N.Y. Acad. Sci., 44, 431 (1943).

19. M. B. Ewing and K. N. Marsh, J. Chem. Therm., 2, 351 (1970).

20. G. Delmas, D. Patterson, and T. Somcynsky, J. Polym. Sci., 57, 7 (1962).

21. C. Watters, H. Daoust, and M. Rinfret, Can. J. Chem., 38, 1087 (1960).

22. P. J. Flory and H. Daoust, J. Polym. Sci., 25 429 (1957).
23. B. E. Eichinger and P. J. Flory, Trans. Faraday Soc., 64, 2061 (1968).

24. C. E. H. Bawn and R. D. Patel, ibid., 52, 1664 (1956).

25. R. W. Kershaw and G. N. Malcolm, ibid., 64, 323 (1968).

26. G. Gee and L. R.G. Treloar, ibid., 38, 147 (1942).

27. G. Gee and W. J. C. Orr, ibid., 42, 507 (1946).

28. B. E. Eichinger and P. J. Flory, ibid., 64, 2035 (1968).

29. W. R. Krigbaum and P. J. Flory, J. Amer. Chem. Soc., 75, 5254 (1953).

30. B. E. Eichinger and P. J. Flory, Trans. Faraday Soc., 64, 2053 (1968).

31. R. S. Jessup, J. Res. Nat. Bur. Stand., A60, 47 (1958). 